\title{
Weyl type Theorems and the Approximate Point Spectrum
}

\author{
M. LAHROUZ AND M. ZOHRY
}

\begin{abstract}
It is shown that, if an operator $T$ on a complex Banach space or its adjoint $T^{*}$ has the single-valued extension property, then the generalized a-Browder's theorem holds for $f(T)$ for every complex-valued analytic function $f$ on a neighborhood of the spectrum of $T$. We also study the generalized a-Weyl's theorem in connection with the single-valued extension property. Finally, we examine the stability of the generalized a-Weyl's theorem under commutative perturbations by finite rank operators.
\end{abstract}

\section{INTRODUCTION}

Throughout this paper $X$ will denote an infinite-dimensional complex Banach space and $\mathcal{L}(X)$ the unital (with unit the identity operator, $I$, on $X)$ Banach algebra of all bounded linear operators acting on $X$. For an operator $T \in \mathcal{L}(X)$, let $T^{*}$ denote its adjoint, $N(T)$ its kernel, $R(T)$ its range, $\sigma(T)$ its spectrum, $\sigma_{a}(T)$ its approximate point spectrum, $\sigma_{s u}(T)$ its surjective spectrum and $\sigma_{p}(T)$ its point spectrum. For a subset $K$ of $\mathbb{C}$ we write iso $(K)$ for its isolated points and $\operatorname{acc}(K)$ for its accumulation points.

From [14] we recall that for $T \in \mathcal{L}(X)$, the ascent $a(T)$ and the descent $d(T)$ are given by

$$
a(T)=\inf \left\{n \geq 0: N\left(T^{n}\right)=N\left(T^{n+1}\right)\right\}
$$

and

$$
d(T)=\inf \left\{n \geq 0: R\left(T^{n}\right)=R\left(T^{n+1}\right)\right\},
$$

2000 Mathematics Subject Classification. 47A53, 47A55.

Key words and phrases. Single-valued extension property, Fredholm theory, generalized a-Weyl's theorem, generalized a-Browder's theorem. 
respectively; the infimum over the empty set is taken to be $\infty$. If the ascent and the descent of $T \in \mathcal{L}(X)$ are both finite then $a(T)=$ $d(T)=p, X=N\left(T^{p}\right) \oplus R\left(T^{p}\right)$ and $R\left(T^{p}\right)$ is closed.

For $T \in \mathcal{L}(X)$ we will denote by $\alpha(T)$ the nullity of $\mathrm{T}$ and by $\beta(T)$ the defect of $T$. If the range $R(T)$ of $T$ is closed and $\alpha(T)<\infty$ (resp., $\beta(T)<\infty$ ) then $T$ is called an upper semi-Fredholm (resp., a lower semi-Fredholm) operator. If $T \in \mathcal{L}(X)$ is either upper or lower semi-Fredholm, then $T$ is called a semi-Fredholm operator, and the index of $T$ is defined by $\operatorname{ind}(T)=\alpha(T)-\beta(T)$. If both $\alpha(T)$ and $\beta(T)$ are finite then $T$ is called a Fredholm operator. For a $T$-invariant closed linear subspace $Y$ of $X$, let $T \mid Y$ denote the operator given by the restriction of $T$ to $Y$.

For a bounded linear operator $T$ and for each integer $n$, define $T_{n}$ to be the restriction of $T$ to $R\left(T^{n}\right)$ viewed as a map from $R\left(T^{n}\right)$ into itself. If for some integer $n$ the range space $R\left(T^{n}\right)$ is closed and $T_{n}=T \mid R\left(T^{n}\right)$ is an upper (resp., lower) semi-Fredholm operator then $T$ is called an upper (resp., lower) semi-B-Fredholm operator. Moreover if $T_{n}$ is a Fredholm operator, then $T$ is called a B-Fredholm operator. In this situation, from [1, Proposition 2.1], $T_{m}$ is a Fredholm operator and $\operatorname{ind}\left(T_{m}\right)=\operatorname{ind}\left(T_{n}\right)$ for each $m \geq n$ which permits to define the index of a B-Fredholm operator $T$ as the index of the Fredholm operator $T_{n}$ where $n$ is any integer such that $R\left(T^{n}\right)$ is closed and $T_{n}$ is a Fredholm operator. Let $B F(X)$ be the class of all B-Fredholm operators and $\rho_{B F}(T)=\{\lambda \in \mathbb{C}: T-\lambda I \in B F(X)\}$ be the B-Fredholm resolvent of $T$ and let $\sigma_{B F}(T)=\mathbb{C} \backslash \rho_{B F}(T)$ the B-Fredholm spectrum of $T$. The class $B F(X)$ has been studied by M. Berkani (see [1, Theorem 2.7]), where it was shown that $T \in \mathcal{L}(X)$ is a B-Fredholm operator if and only if $T=T_{0} \oplus T_{1}$ where $T_{0}$ is a Fredholm operator and $T_{1}$ is a nilpotent one. He also proved that $\sigma_{B F}(T)$ is a closed subset of $\mathbb{C}$ and showed that the spectral mapping theorem holds for $\sigma_{B F}(T)$, that is, $f\left(\sigma_{B F}(T)\right)=\sigma_{B F}(f(T))$ for any complex-valued analytic function on a neighborhood of the spectrum $\sigma(T)$.

An operator $T \in \mathcal{L}(X)$ is called a Weyl operator if it is Fredholm of index 0, a Browder operator if it is Fredholm of finite ascent and descent and a B-Weyl operator if it is B-Fredholm of index 0. The Weyl spectrum, the Browder spectrum and the B-Weyl spectrum of $T$ are defined by

$$
\sigma_{w}(T)=\{\lambda \in \mathbb{C}: T-\lambda I \text { is not Weyl }\}
$$




$$
\begin{gathered}
\sigma_{b}(T)=\{\lambda \in \mathbb{C}: T-\lambda I \text { is not Browder }\}, \\
\sigma_{B W}(T)=\{\lambda \in \mathbb{C}: T-\lambda I \text { is not B-Weyl }\},
\end{gathered}
$$

respectively. We will denote by $E(T)$ (resp. $E^{a}(T)$ ) the set of all eigenvalues of $T$ which are isolated in $\sigma(T)$ (resp., $\sigma_{a}(T)$ ) and by $E_{0}(T)$ (resp. $E_{0}^{a}(T)$ ) the set of all eigenvalues of $T$ of finite multiplicity which are isolated in $\sigma(T)$ (resp., $\sigma_{a}(T)$ ).

Let $S F(X)$ be the class of all semi-Fredholm operators on $X$, $S F_{+}(X)$ the class of all upper semi-Fredholm operators on $X$ and $S F_{+}^{-}(X)$ the class of all $T \in S F_{+}(X)$ such that $\operatorname{ind}(T) \leq 0$. For $T \in \mathcal{L}(X)$, let

$$
\begin{aligned}
\sigma_{S F}(T) & =\{\lambda \in \mathbb{C}: T-\lambda I \notin S F(X)\}, \\
\sigma_{S F_{+}^{-}}(T) & =\left\{\lambda \in \mathbb{C}: T-\lambda I \notin S F_{+}^{-}(X)\right\},
\end{aligned}
$$

$\rho_{S F}(T)=\mathbb{C} \backslash \sigma_{S F}(T)$ and $\rho_{S F_{+}^{-}}(T)=\mathbb{C} \backslash \sigma_{S F_{+}^{-}}(T)$.

Similarly, let $S B F(X)$ be the class of all semi-B-Fredholm operators on $\mathrm{X}, S B F_{+}(X)$ the class of all upper semi-B-Fredholm operators on $X$ and $S B F_{+}^{-}(X)$ the class of all $T \in S B F_{+}(X)$ such that $\operatorname{ind}(T) \leq 0$. For $T \in \mathcal{L}(X)$, the sets $\sigma_{S B F}(T), \rho_{S B F}(T), \sigma_{S B F_{+}^{-}}(T)$ and $\rho_{S B F_{+}^{-}}(T)$ are defined in an obvious way.

An operator $T \in \mathcal{L}(X)$ is called semi-regular if $R(T)$ is closed and $N(T) \subseteq R\left(T^{n}\right)$ for every $n \in \mathbb{N}$. The semi-regular resolvent set is defined by s-reg $(T)=\{\lambda \in \mathbb{C}: T-\lambda I$ is semi- regular $\}$, we note that s-reg $(T)=\operatorname{s-reg}\left(T^{*}\right)$ is an open subset of $\mathbb{C}$. As a consequence of [8, Théorème 2.7], we obtain the following result.

Proposition 1.1. Let $T \in \mathcal{L}(X)$.

(i) If $T$ has the $S V E P$ then $s-\operatorname{reg}(T)=\rho_{a}(T)=\mathbb{C} \backslash \sigma_{a}(T)$.

(ii) If $T^{*}$ has the $S V E P$ then $s$-reg $(T)=\rho_{s u}(T)=\mathbb{C} \backslash \sigma_{s u}(T)$.

We recall that an operator $T \in \mathcal{L}(X)$ has the single-valued extension property, abbreviated SVEP, if, for every open set $U \subset \mathbb{C}$, the only analytic solution $f: U \longrightarrow X$ of the equation $(T-\lambda I) f(\lambda)=0$ for all $\lambda \in U$ is the zero function on $U$. We will denote by $\mathcal{H}(\sigma(T))$ the set of all complex-valued functions which are analytic on an open set containing $\sigma(T)$.

The remainder of the following deals with Riesz points and left poles. A complex number $\lambda$ is said to be Riesz point of $T \in \mathcal{L}(X)$ if $\lambda \in \operatorname{iso}(\sigma(T))$ and the corresponding spectral projection is of finitedimensional range. The set of all Riesz points of $T$ will be denoted by 
$\Pi_{0}(T)$. It is known that if $T \in \mathcal{L}(X)$ and $\lambda \in \sigma(T)$, then $\lambda \in \Pi_{0}(T)$ if and only if $T-\lambda I$ is Fredholm of finite ascent and descent (see [3]). Consequently $\sigma_{b}(T)=\sigma(T) \backslash \Pi_{0}(T)$. We will denote by $\Pi(T)$ the set of all poles of the resolvent of $T$. A complex number $\lambda \in \sigma_{a}(T)$ is said to be a left pole of $T$ if $a(T-\lambda I)<\infty$ and $R\left((T-\lambda I)^{a(T-\lambda I)+1}\right)$ is closed, and that it is a left pole of $T$ of finite rank if it is a left pole of $T$ and $\alpha(T-\lambda I)<\infty$. We will denote by $\Pi^{a}(T)$ the set of all left poles of $T$, and by $\Pi_{0}^{a}(T)$ the set of all left poles of $T$ of finite rank. If $\lambda \in \Pi^{a}(T)$, then it is easily seen that $T-\lambda I$ is an operator of topological uniform descent, therefore from [4], it follows that $\lambda$ is isolated in $\sigma_{a}(T)$ [2, Theorem 2.5]. Let $T \in \mathcal{L}(X)$ and $\lambda \in \mathbb{C}$ be isolated in $\sigma_{a}(T)$; then $\lambda \in \Pi^{a}(T)$ if and only if $\lambda \notin \sigma_{S B F_{+}^{-}}(T)$, and $\lambda \in \Pi_{0}^{a}(T)$ if and only if $\lambda \notin \sigma_{S F_{+}^{-}}(T)$.

For $T \in \mathcal{L}(X)$ we will say that:

(i) $T$ satisfies Weyl's theorem if $\sigma_{w}(T)=\sigma(T) \backslash E_{0}(T)$;

(ii) $T$ satisfies generalized Weyl's theorem if

$$
\sigma_{B W}(T)=\sigma(T) \backslash E(T) ;
$$

(iii) $T$ satisfies a-Weyl's theorem if

$$
\sigma_{S F_{+}^{-}}(T)=\sigma_{a}(T) \backslash E_{0}^{a}(T) ;
$$

(iv) $T$ satisfies generalized a-Weyl's theorem if

$$
\sigma_{S B F_{+}^{-}}(T)=\sigma_{a}(T) \backslash E^{a}(T) ;
$$

(v) $T$ satisfies Browder's theorem if

$$
\sigma_{w}(T)=\sigma(T) \backslash \Pi_{0}(T) ;
$$

(vi) $T$ satisfies generalized Browder's theorem if

$$
\sigma_{B W}(T)=\sigma(T) \backslash \Pi(T) ;
$$

(vii) $T$ satisfies a-Browder's theorem if

$$
\sigma_{S F_{+}^{-}}(T)=\sigma_{a}(T) \backslash \Pi_{0}^{a}(T) ;
$$

(viii) $T$ satisfies generalized a-Browder's theorem if

$$
\sigma_{S B F_{+}^{-}}(T)=\sigma_{a}(T) \backslash \Pi^{a}(T) .
$$

Before proving our main result we deal with some preliminary results. 
Proposition 1.2. Let $T \in \mathcal{L}(X)$.

(i) If $T$ has the $S V E P$ then $\operatorname{ind}(T-\lambda I) \leq 0$ for every $\lambda \in$ $\rho_{S B F}(T)$.

(ii) If $T^{*}$ has the $S V E P$ then $\operatorname{ind}(T-\lambda I) \geq 0$ for every $\lambda \in$ $\rho_{S B F}(T)$.

Proof. (i) Let $\lambda \in \rho_{S B F}(T)$, then there exists an integer $p$ such that $\left(T \mid R(T-\lambda I)^{p}\right)-\lambda I=(T-\lambda I) \mid R(T-\lambda I)^{p}$ is semi-Fredholm. From the Kato decomposition, there exists $\delta>0$ such that

$$
\{\lambda \in \mathbb{C}: 0<|\mu-\lambda|<\delta\} \subseteq \operatorname{s-reg}\left(T \mid R(T-\lambda I)^{p}\right) .
$$

Since $T$ has the SVEP, Proposition 1.1 implies that

$$
\text { s-reg }\left(T \mid R(T-\lambda I)^{p}\right)=\rho_{a}\left(T \mid R(T-\lambda I)^{p}\right) .
$$

Therefore, $N\left(\left(T \mid R(T-\lambda I)^{p}\right)-\mu I\right)=0$ and so $\operatorname{ind}(T-\mu I)=$ $\operatorname{ind}\left(\left(T \mid R(T-\lambda I)^{p}-\mu I\right) \leq 0\right.$, holding for $0<|\mu-\lambda|<\delta$. Thus, by the continuity of the index we obtain $\operatorname{ind}(T-\lambda) \leq 0$.

(ii) Follows by similar reasoning, and may also be derived from the first assertion and the fact that ind $\left(T^{*}\right)=-\operatorname{ind}(T)$.

Corollary 1.3. Let $T$ be a bounded linear operator on $X$. If $T^{*}$ has the SVEP, then $\sigma_{S F_{+}^{-}}(T)=\sigma_{w}(T)$.

Proof. We have only to show that $\sigma_{w}(T) \subseteq \sigma_{S F_{+}^{-}}(T)$, since the other inclusion is always verified. Let $\lambda$ be given in $\rho_{S F_{+}^{-}}(T)$, then $T-\lambda I$ is semi-Fredholm and ind $(T-\lambda I) \leq 0$. Since $T^{*}$ has the SVEP, Proposition 1.2 implies that ind $(T-\lambda I) \geq 0$, and hence ind $(T-\lambda I)=0$, which proves that $T-\lambda I$ is Fredholm of index 0 and $\lambda \in \rho_{w}(T)$.

The following results relate the generalized a-Weyl's theorem and the generalized a-Browder's theorem to the single-valued extension property. As motivation for the proofs, we use some ideas in [10,12].

Proposition 1.4. Let $T$ be a bounded linear operator on $X$.

(i) If $T^{*}$ has the SVEP, then T satisfies generalized a-Weyl's theorem if and only if it satisfies generalized Weyl's theorem.

(ii) If $T$ has the SVEP, then $T^{*}$ satisfies generalized a-Weyl's theorem if and only if it satisfies generalized Weyl's theorem.

Proof. (i) Since $T^{*}$ has the SVEP, [6, Proposition 1.3.2] implies that $\sigma(T)=\sigma_{a}(T)$ and consequently $E^{a}(T)=E(T)$. Suppose that $T$ satisfies generalized Weyl's theorem, then $\sigma_{B W}(T)=\sigma(T) \backslash E(T)=$ 
$\sigma_{a}(T) \backslash E^{a}(T)$. Let $\lambda \notin \sigma_{S B F^{-}}(T)$ be given, then $T-\lambda I$ is semiB-Fredholm and ind $(T-\lambda I) \leq 0$. Therefore, by Proposition 1.2, it follows that ind $(T-\lambda I)=0$ and consequently $T-\lambda I$ is B-Fredholm of index 0. Hence $\lambda \notin \sigma_{B W}(T)$ and $\sigma_{B W}(T) \subset \sigma_{S B F_{+}^{-}}(T)$. Since the opposite inclusion is clear, we conclude that indeed $\sigma_{S B F_{+}^{-}}(T)=$ $\sigma_{B W}(T)=\sigma_{a}(T) \backslash E^{a}(T)$ which proves the equivalence between generalized Weyl's theorem and generalized a-Weyl's theorem for $T$.

(ii) Similar to the proof of the first assertion.

Our main result reads now as follows.

Theorem 1.5. Let $T$ be a bounded linear operator on $X$. If $T$ or its adjoint $T^{*}$ satisfies the $S V E P$, then generalized a-Browder's theorem holds for $f(T)$ for every $f \in H(\sigma(T))$.

Proof. Let us establish that generalized a-Browder's theorem holds for $T$. If $T^{*}$ has the SVEP, then by [12, Theorem 2.4], it follows that a-Browder's theorem holds for $T$, and consequently Browder's theorem holds for $T$. Thus $\sigma_{S F_{+}^{-}}(T)=\sigma_{a}(T) \backslash \Pi_{0}^{a}(T)$ and $\sigma_{b}(T)=$ $\sigma(T) \backslash \Pi_{0}(T)$. Moreover, since $\sigma_{a}(T)=\sigma(T), \Pi_{0}^{a}(T)=\Pi_{0}(T)$, it follows that $\sigma_{S F_{+}^{-}}(T)=\sigma(T) \backslash \Pi_{0}(T)$. Because $\sigma_{S F_{+}^{-}}(T)=\sigma_{w}(T)$, see Corollary 1.3, it follows that $\sigma_{S F_{+}^{-}}(T)=\sigma(T) \backslash \Pi_{0}(T)=\sigma_{w}(T)=$ $\sigma_{b}(T)$. Let $\lambda \in \Pi^{a}(T)$ be given; then $\lambda$ is isolated in $\sigma_{a}(T)$ and by $\left[2\right.$, Theorem 2.8], it follows that $\lambda \notin \sigma_{S B F_{+}^{-}}(T)$ which shows that $\Pi^{a}(T) \subseteq \sigma_{a}(T) \backslash \sigma_{S B F_{+}^{-}}(T)$. Conversely if $\lambda \in \sigma_{a}(T) \backslash \sigma_{S B F_{+}^{-}}(T)$, then $T-\lambda I$ is semi-B-Fredholm and ind $(T-\lambda I) \leq 0$. Then, since $T^{*}$ has the SVEP, Proposition 1.2 gives ind $(T-\lambda I)=0$. Therefore $T-$ $\lambda I$ is Fredholm and $\lambda \notin \sigma_{w}(T)=\sigma_{b}(T)$ which shows that $\lambda \in \Pi_{0}(T)$. Consequently $\lambda$ is isolated in $\sigma_{a}(T)$ and hence $\lambda \in \Pi^{a}(T)$. Thus $\sigma_{a}(T) \backslash \sigma_{S B F_{+}^{-}}(T) \subset \Pi^{a}(T)$ and generalized a-Browder's theorem holds for $T$. Now if $T$ has the SVEP, let $\lambda \in \sigma_{a}(T) \backslash \sigma_{S B F_{+}^{-}}(T)$; $\lambda \in \rho_{S B F_{+}^{-}}(T)$, then there exists an integer $p$ such that $R(T-\lambda I)^{p}$ is closed and $\left(T \mid R(T-\lambda I)^{p}\right)-\lambda I=(T-\lambda I) \mid R(T-\lambda I)^{p}$ is a semi-Fredholm operator. Then, by the Kato decomposition, there exists $\delta>0$ for which

$$
\begin{aligned}
\{\mu \in \mathbb{C}: 0<|\mu-\lambda|<\delta\} & \\
& \subseteq \operatorname{s-reg}\left(T \mid R(T-\lambda I)^{p}\right) \cap \rho_{S F}\left(T \mid R(T-\lambda I)^{p}\right) .
\end{aligned}
$$


Since $T$ has the SVEP, so does $T \mid R(T-\lambda I)^{p}$. Therefore

$$
\operatorname{s-reg}\left(T \mid R(T-\lambda I)^{p}\right)=\rho_{a}\left(T \mid R(T-\lambda I)^{p}\right)
$$

and

$$
\begin{aligned}
\{\mu \in \mathbb{C}: 0<\mid \mu & -\lambda \mid<\delta\} \\
& \subseteq \rho_{a}\left(T \mid R(T-\lambda I)^{p}\right) \cap \rho_{S F}\left(T \mid R(T-\lambda)^{p}\right),
\end{aligned}
$$

hence $\lambda \in \operatorname{iso}\left(\sigma_{a}(T) \cap \rho_{S B F}(T)\right)$. By [2, Theorem 2.8], it follows that $\lambda \in \Pi_{a}(T)$ and $\sigma_{a}(T) \backslash \sigma_{S B F_{+}^{-}}(T) \subset \Pi^{a}(T)$. Since the other inclusion is clear we get $\sigma_{a}(T) \backslash \sigma_{S B F_{+}^{-}}(T)=\Pi_{a}(T)$ and thus generalized aBrowder's theorem holds for $T$. Finally, if $f \in H(\sigma(T))$, by [6, Theorem 3.3.6] $f(T)$ or $f\left(T^{*}\right)$ satisfies the SVEP and the above argument implies that generalized a-Browder's theorem holds for $f(T)$.

From Theorem 1.5 we obtain the following useful consequence.

Corollary 1.6. Let $T$ be a bounded linear operator on $X$. If $T$ or $T^{*}$ has the SVEP then generalized a-Weyl's theorem holds for $T$ if and only if $E^{a}(T)=\Pi^{a}(T)$.

Proof. We only have to use the fact that an operator $T$ satisfying generalized a-Browder's theorem, satisfies generalized a-Weyl's theorem if and only if $\Pi^{a}(T)=E^{a}(T)$.

In [7] the class of the operators $T \in \mathcal{L}(X)$ for which $K(T)=\{0\}$ was studied and it was shown that for such operators, the spectrum is connected and the single-valued extension property is satisfied.

Proposition 1.7. Let $T \in \mathcal{L}(X)$. If there exists a complex number $\lambda$ for which $K(T-\lambda I)=\{0\}$ then $f(T)$ satisfies generalized aBrowder's theorem for every $f \in \mathcal{H}(\sigma(T))$. Moreover, if in addition, $N(T-\lambda I)=\{0\}$, then generalized a-Weyl's theorem holds for $f(T)$ for any $f \in \mathcal{H}(\sigma(T))$.

Proof. Let $f$ be a non-constant complex-valued analytic function on an open neighborhood of $\sigma(T)$. Since $T$ has the SVEP so does $f(T)$ and by Theorem 1.5 generalized a-Browder's theorem holds for $f(T)$. Now assume that $N(T-\lambda I)=\{0\}$ and $\beta \in \sigma(f(T))$ then $f(z)-\beta I=P(z) g(z)$ where $g$ is complex-valued analytic function on a neighborhood of $\sigma(T)$ without any zeros in $\sigma(T)$ while $P$ is a 
complex polynomial of the form $P(z)=\prod_{i=1}^{n}\left(z-\lambda_{i}\right)^{p_{i}}$ with distinct roots $\lambda_{1}, \ldots, \lambda_{n} \in \sigma(T)$. Since $g(T)$ is invertible, we have

$$
N(f(T)-\beta I)=N(P(T))=\oplus_{i=1}^{n} N\left(T-\lambda_{i} I\right)^{p_{i}} .
$$

On the other hand, [7, Proposition 2.1] ensures that $\sigma_{p}(T) \subseteq\{\lambda\}$ and since $T-\lambda I$ is injective, we deduce that $\sigma_{p}(T)=\emptyset$. Consequently $N(f(T)-\beta I)=\{0\}$ which proves that $\sigma_{p}(f(T))=\emptyset$. Thus $E^{a}(f(T))=\Pi^{a}(f(T))=\emptyset$ and generalized a-Weyl's theorem holds for $f(T)$.

Proposition 1.8. Let $T$ be a bounded linear operator on $X$ satisfying the SVEP. If $T-\lambda I$ has finite descent at every $\lambda \in E^{a}(T)$, then $T$ obeys generalized a-Weyl's theorem.

Proof. Let $\lambda \in E^{a}(T)$, then $p=d(T-\lambda I)<\infty$ and since $T$ has the SVEP it follows (see [13, Proposition 3]) that $a(T-\lambda I)=d(T-$ $\lambda I)=p$ and by [5, Satz 101.2], $\lambda$ is a pole of the resolvent of $T$ or order $p$, consequently $\lambda$ is an isolated point in $\sigma_{a}(T)$. Then $X=$ $K(T-\lambda I) \oplus H_{0}(T-\lambda I)$, with $K(T-\lambda I)=R(T-\lambda I)^{p}$ is closed, therefore $\lambda \in \Pi^{a}(T)$.

Now let us consider the class $\mathcal{P}(X)$ defined as those operators $T \in \mathcal{L}(X)$ for which for every complex number $\lambda$ there exists a positive integer $p_{\lambda}$ such that $H_{0}(T-\lambda I)=N(T-\lambda I)^{p_{\lambda}}$. This class has been introduced and studied in [10,11], it was shown that it contains every M-hyponormal, log-hyponormal, p-hyponormal and totally paranormal operator. It was also established that the SVEP is shared by all the operators lying in $\mathcal{P}(X)$ and generalized Weyl's theorem holds for $f(T)$ whenever $T \in \mathcal{P}(X)$ and $f \in \mathcal{H}(\sigma(T))$.

Proposition 1.9. Let $T \in \mathcal{P}(X)$ be such that $\sigma(T)=\sigma_{a}(T)$ then generalized a-Weyl's theorem holds for $f(T)$ for every $f \in \mathcal{H}(\sigma(T))$.

Proof. By the spectral mapping theorem for the spectrum and the approximate point spectrum, and the fact that $f(T) \in \mathcal{P}(X)$, it suffices to establish generalized a-Weyl's theorem for $T$. Since $\sigma(T)=$ $\sigma_{a}(T)$ it follows that

$$
E^{a}(T)=\sigma_{p}(T) \cap \operatorname{iso}\left(\sigma_{a}(T)\right)=\sigma_{p}(T) \cap \operatorname{iso}(\sigma(T))=E(T) .
$$

Let $\lambda \in E^{a}(T)=E(T)$, then $X=H_{0}(T-\lambda I) \oplus K(T-\lambda I)$ and $K(T-\lambda I)$ is closed. Since $T \in \mathcal{P}(X)$, let $p_{\lambda}$ be a positive integer 
for which $H_{0}(T-\lambda I)=N(T-\lambda I)^{p_{\lambda}}$, therefore

$$
\begin{aligned}
R(T-\lambda I)^{p_{\lambda}} & =(T-\lambda I)^{p_{\lambda}}\left(H_{0}(T-\lambda I) \oplus K(T-\lambda I)\right) \\
& =(T-\lambda I)^{p_{\lambda}}(K(T-\lambda I)) \\
& =K(T-\lambda I),
\end{aligned}
$$

thus $R(T-\lambda I)^{p_{\lambda}}=R(T-\lambda I)^{p_{\lambda}+1}$ which by Proposition 1.8 shows that the operator $T$ obeys generalized a-Weyl's theorem.

\section{Generalized a-Weyl's Theorem and Perturbation}

In general, we cannot expect that generalized a-Browder's theorem necessarily holds under finite rank perturbations. However, it does hold under commutative ones, as the following result shows.

Theorem 2.1. [2, Theorem 3.2] If $T \in \mathcal{L}(X)$ is an operator satisfying generalized a-Browder's theorem and $F$ is a finite rank operator such that $T F=F T$ then $T+F$ satisfies generalized a-Browder's theorem.

Lemma 2.2. Let $T \in \mathcal{L}(X)$ be an injective operator. If $F$ is a finite rank operator on $X$ such that $F T=T F$, then $R(F) \subseteq R(T)$.

Proof. Since $F$ is a finite rank operator on $X$ there exist two systems: a system of linearly independent vectors $e_{i}$ for $i=1, \ldots, n$ and a system of non-zero bounded linear functionals $f_{i}$ for $i=1, \ldots, n$ on $X$ such that

$$
F(x)=\sum_{i=1}^{n} f_{i}(x) e_{i} \quad(x \in X) .
$$

Moreover, we have

$$
\sum_{i=1}^{n} f_{i}(x) T e_{i}=T F(x)=F T(x)=\sum_{i=1}^{n} f_{i}(T x) e_{i} \quad(x \in X) .
$$

On the other hand, since $T$ is injective, it is clear that the vectors $T e_{i}(1 \leq i \leq n)$ are linearly independent. Hence $F(x) \in$ $\operatorname{Vect}\left(\left\{e_{1}, \cdots, e_{n}\right\}\right)=\operatorname{Vect}\left(\left\{T e_{1}, \cdots, T e_{n}\right\}\right)$ for all $x \in X$. Thus $R(F) \subseteq R(T)$, as desired.

Lemma 2.3. Let $T \in \mathcal{L}(X)$. If $F$ is a finite rank operator on $X$ such that $F T=T F$ then $\lambda \in \operatorname{acc}\left(\sigma_{a}(T)\right)$ if and only if $\lambda \in \operatorname{acc}\left(\sigma_{a}(T+F)\right)$. 
Proof. Let $\lambda \notin \operatorname{acc}\left(\sigma_{a}(T)\right)$ be given, there exists $\delta>0$ such that if $0<|\mu-\lambda|<\delta$ then $\alpha(T-\mu I)=0$ and $R(T-\mu I)$ is closed. This gives us a bounded linear operator $S: R(T-\mu I) \longrightarrow X$ such that $S(T-\mu I)=I$ and $(T-\mu I) S=I \mid R(T-\mu I)$. To see that $\lambda \notin \operatorname{acc}\left(\sigma_{a}(T+F)\right)$, suppose that $\mu \in \sigma_{a}(T+F)$, and choose unit vectors $x_{n} \in X$ such that $(T+F-\mu I) x_{n} \rightarrow 0$ as $n \rightarrow \infty$. Let $\left(x_{n(k)}\right)_{k}$ be a subsequence such that $F x_{n(k)} \rightarrow x \in R(F)$ as $k \rightarrow \infty$, and since this level of generality is not needed here, we may assume that $F x_{n} \rightarrow x$ as $n \rightarrow \infty$. Therefore $S(T+F-\mu I) x_{n}=x_{n}+S F x_{n} \rightarrow 0$ as $n \rightarrow \infty$, and since $\lim S F x_{n}=S x$ exists, it follows that $\lim x_{n}=$ $-S x$ and consequently $x \neq 0$. Next observe that $x=\lim F x_{n}=$ $-F S x \in R(F)$, then since Lemma 2.2 asserts that $R(F) \subseteq R(T)$, we obtain $(T-\mu I) x=-(T-\mu I) F S x=-F(T-\mu I) S x=-F x$, hence $(T+F-\mu I) x=0$. Thus $\mu \in \sigma_{p}(T+F)$. Finally, because eigenvectors corresponding to distinct eigenvalues of an operator are linearly independent, and since all the eigenvectors of $T+F$ belong to the finite dimensional subspace $R(F)$, it follows that $\sigma_{a}(T+F)$ may contain only finitely many points $\mu$ such that $0<|\mu-\lambda|<\delta$, and consequently $\lambda \notin \operatorname{acc}\left(\sigma_{a}(T+F)\right)$. The opposite inclusion is similarly obtained.

An operator $T \in \mathcal{L}(X)$ is said to be approximate-isoloid if any isolated point of $\sigma_{a}(T)$ is an eigenvalue of $T$.

Theorem 2.4. Let $T$ be an approximate-isoloid operator on $X$ that satisfies generalized a-Weyl's theorem. If $F$ is an operator of finite rank on $X$ such that $F T=T F$ then $T+F$ satisfies generalized a-Weyl's theorem.

Proof. Since by Theorem 2.1 generalized a-Browder's theorem holds for $T+F$ it suffices, from Corollary 1.5, to prove that $E^{a}(T+F)=$ $\Pi^{a}(T+F)$. Let $\lambda \in E^{a}(T+F)$ be given, then $\lambda \in \operatorname{iso}\left(\sigma_{a}(T+F)\right)$ and $\lambda \in \sigma_{p}(T+F)$, hence $\lambda \notin \operatorname{acc}\left(\sigma_{a}(T+F)\right.$ and by Lemma 2.3 $\lambda \notin \operatorname{acc}\left(\sigma_{a}(T)\right.$. We distinguish two cases. Firstly if $\lambda \notin \sigma_{a}(T)$, $T-\lambda I$ is injective with a closed range and $T-\lambda I$ is an upper semiFredholm operator on $X$ such that $\operatorname{ind}(T-\lambda I) \leq 0$, and since $F$ is a finite rank operator on $X$, it follows that $T+F-\lambda I$ is an upper semi-Fredholm operator and $\operatorname{ind}(T+F-\lambda I)=\operatorname{ind}(T-\lambda I) \leq 0$. Then $\lambda \notin \sigma_{S F_{+}^{-}}(T+F)$ and $\lambda \in \Pi^{a}(T+F)$. On the other hand if $\lambda \in \sigma_{a}(T)$, then $\lambda \in \operatorname{iso}\left(\sigma_{a}(T)\right)$ and since $T$ is approximate-isoloid $\lambda \in \sigma_{p}(T)$. Thus $\lambda \in \operatorname{iso}\left(\sigma_{a}(T)\right) \cap \sigma_{p}(T)=E^{a}(T)$. From the 
fact that $T$ obeys generalized a-Weyl's theorem, it follows that $\lambda \notin$ $\sigma_{S B F_{+}^{-}}(T)=\sigma_{S B F_{+}^{-}}(T+F)$ and since $\lambda \in \operatorname{iso}\left(\sigma_{a}(T+F)\right)$, it follows that $\lambda \in \Pi^{a}(T+F)$. Finally $E^{a}(T+F) \subset \Pi^{a}(T+F)$, and since the reverse inclusion is verified, $T+F$ obeys generalized a-Weyl's theorem.

\section{REFERENCES}

[1] M. Berkani, On a class of quasi-Fredholm operators, Int. Eq. Oper. Th. 34 (1999), 244-249.

[2] M. Berkani and J. J. Koliha, Weyl type theorems for bounded linear operators, Acta Sci. Math. (Szeged) 69 (2003), 359-376.

[3] S. R. Caradus, W. E. Pfaffenberger, B. Yood, Calkin algebras and algebras of operators on Banach spaces, Marcel Dekker, New York, 1974.

[4] S. Grabiner, Uniform ascent and descent of bounded operators, J. Math. Soc. Japan 34 (1982), 317-337.

[5] H. Heuser, Funktionalanalysis, 2nd ed., Teubner, Stuttgart, 1986.

[6] K. B. Laursen and M. M. Neumann, An introduction to local spectral theory, LMS Monographs 20, Clarendon Press, Oxford, 2000.

[7] M. Mbekhta, Sur la théorie spectrale locale et limite des nilpotents, Proc. Amer. Math. Soc. 110 (1990), 621-631.

[8] M. Mbekhta and A. Ouahab, Opérateur s-régulier dans un espace de Banach et théorie spectrale, Acta Sci. Math. (Szeged) 59 (1994), 525-543.

[9] M. Mbekhta and V. Müller, On the axiomatic theory of spectrum II, Studia Math. 119 (1996), 129-147.

[10] M. Oudghiri, Sur le théorème de Weyl, Thèse en cotutelle (Université Abdelmalek Essaadi, Tétouan, et Université des Sciences et Technologies, Lille), (2004).

[11] M. Oudghiri, Weyl's and Browder's theorem for operators satisfying the SVEP, Studia Math. 163 (2004), 85-101.

[12] M. Oudghiri, Weyl's theorem and perturbations, preprint.

[13] C. Schmoeger, On isolated points of the spectrum of bounded linear operators, Proc. Amer. Math. Soc. 117 (1993), 715-719.

[14] A. Taylor and D. Lay, Introduction to functional analysis, 2nd ed., John Wiley \& Sons, 1980.

M. Lahrouz, M. Zohry,

Université Abdelmalek Essaadi,

Faculté des Sciences,

Departement de Mathématique,

BP 2121 Tétouan, Moroc

zohry@fst.ac.ma

Received on 22 February 2005. 